\title{
MAP BASED INTRA-CELL METHOD FOR LOCATION PREDICTION OVER UMTS NETWORK PLATFORM
}

\section{Abo-Zahhad, Sabah M. Ahmed and M. Mourad}

\author{
Electrical and Electronics Eng. Department, Faculty of Engineering, Assiut University, \\ Assiut, Egypt (zahhad@yahoo.com)
}

(Received June 16, 2011 Accepted August 2, 2011)

Location based services (LBS) and their applications are regarded as the most growing services applied on communication systems. According to the great enhancement in the mobile network platform and its related database servers, we are not only able to develop LBS applications but also to enhance the performance of mobile networks. Future location prediction of mobile user results in great enhancement in LBS applications and mobile network performance. Many researches were introduced to predict location of mobile user. In [7] User Mobility Pattern (UMP) algorithm based on sequential mining prediction has been developed to deal with future location prediction in mobile networks. The main limitations of this pattern are: map based intra cell prediction cannot be extracted and the timestamps aren't associated with rules. In [8], another method has been introduced using All frequent Movement Pattern (ALLMOP) algorithm for extracting all frequent movement patterns within movement Rule based Location Prediction method (RLP). RLP method works on GPS network map based platform without manipulating cell to cell movement. So, it can't be used to enhance mobile network's resource allocation and mobility management. In this paper we proposed a new method, Intra Cell Movement Prediction (ICMP), for mobile user's future location prediction on Universal Mobile Telecommunication System (UMTS) environments to benefit from both intra and inter cell based techniques for both network and services enhancement. The proposed method depends on map based intra-cell prediction and utilizes the network database and positioning techniques in extracting user trajectories and movement rules. As a result, the user position can be predicted for the next movement step. The performance of the proposed algorithm is evaluated through computer simulation and compared with that of [7] and [8]. The simulation results indicate that the proposed method is highly efficient for both network and services' improvements as it is used to enhance location based services with satisfactory accuracy, network's resource utilization, reduce the latency in accessing resources and mobility management.

\section{Introduction}

According to the increase in the number of mobile users all over the world, applications depending on mobile networks become with appreciated and valuable consideration in human life. LBS can be defined as an information and entertainment service, accessible with mobile devices through the mobile network and utilizing the 
ability to use the geographical position of mobile subscriber. Current LBSs use information about current locations of users to provide needed services. Although the common computing strategy in LBSs benefits the users, there are additional benefits when future locations are predicted. One major advantage of location prediction is that it provides LBSs with extended resources, mainly time, to improve system reliability which in turn increases the users' confidence and the demand for LBSs. A lot of algorithms and techniques were developed to predict future locations in mobile networks [7]-[8]. As a result of obvious improvements in mobile network platform and its services starting from first generation till now we will stop at UMTS platform which introduces high quality data, services, high users' capacity and availability. Utilizing UMTS advantages and applying position detection techniques such as Angle of Arrival (AOA), Time Difference of Arrival (TDOA), Enhanced-Observed Time Difference (EOTD) and Uplink Time Difference of Arrival (U-TDOA) result in improvement in the accuracy of location detection. In [10], it has been shown that the most accurate position detection method is the U-TDOA with network configuration shown in Figure 1. Moreover, sectoring technique is used to increase the system capacity as well as the coverage in UMTS networks. In sectoring technique the number of sectors belonging to the site is increased in order to increase the capacity of the cellular system. Directional antennas used in sectoring techniques transmit only to those directions where the signal is really intended. This results in cutting down co-channel interference and allowing a network to serve more subscribers. This offers more capacity to the network. By using Location Measurement Units (LMU), the handset location is calculated in location server using time differences of arrival of the signals from each node B at both the handset and the LMU [9]. As a result U-OTDOA technique can be adapted to reach satisfactory positioning accuracy which will be considered as the first step in the trip to get needed location prediction. The second step of location prediction trip utilizes sectoring technique in order to decrease intra cell interference and increase the number of subscribers. So, intra-cell prediction which is adopted here is highly required to enhance sector to sector handover quality and save allocation resources of mobile network.

It should be noticed that UMP algorithm that is based on sequential mining doesn't introduce intra cell prediction [7]. In [8], RLP method has been developed using ALLMOP algorithm and GPS network platform. However, the use of GPS network platform results in no enhancement on mobile network resources allocations and management. In fact, the UMP algorithm is outperformed by the RLP method. The two short comes of [7] and [8] are solved here through the utilization of both mobile network structure and ALLMOP algorithm to create map based intra cell architecture. This yields to enhancement in mobile network resources and location services.

This paper is organized as follows. Section 2 reviews the related publications. Positioning in UMTS network platform is presented in section 3. Section 4 presents future location prediction in mobility management enhancement. The problem definition is discussed in section 5. In section 6, the process of trajectory extracting over organized graph and frequent pattern algorithm are explained. Section 7 introduces the movement rules generation and the next location prediction algorithm. In section 8, the experimental results are given. Finally, the conclusion and future work are presented in section 9 . 


\section{Related work}

In literature many approaches and algorithms have been introduced to predict future location of a mobile user; each has specific requirements and results in different accuracy level [1]-[8]. The sequential pattern mining problem that was discussed in [1] doesn't take into account the network topology while generating the candidate patterns. This is due to considering only the sequence of neighboring cells of the network as a mobility pattern without map category support. So, sequential pattern algorithm cannot be applied directly for mining mobility patterns. A predictive mobility management algorithm for predictive distance-based mobility management for Personal Communication System (PCS) networks computing and communications is discussed in [2]. In order to estimate the future location of a user, a mobile motion prediction (MMP) algorithm has been developed. However, the MMP algorithm is highly sensitive to the user's random movements which decrease the pattern performance. Mobility modeling, location tracking, and trajectory prediction in wireless ATM networks are discussed in [3] where two level schemes are adopted to combine a local with a global prediction models. The top level is the global mobility model (GMM), whose resolution is determined in terms of the cells crossed by a mobile user during the lifetime of the connection. The bottom level is the local mobility model (LMM), whose resolution is determined in terms of three sample space (speed, direction, position) that varies with time. In [3] LMM is used to model the intra-cell movements of the mobile users. On the other hand, GMM is used to predict the inter-cell movement trajectory of a user by matching the user's actual path to one of the existing mobility patterns. For this purpose, pattern matching techniques are adopted. However, the weakness of the technique presented in [3] is revealed at this point because discovering the mobility patterns cannot be achieved. This problem is solved in the proposed algorithm by considering the dependence of the mobility pattern on the previous movement records. In [4] a mobile user's current velocity and location is correlated in time based on the Gauss-Markov model. In this model a mobile user's future location is predicted by the network based on the information gathered from the user's last report of location and velocity.

GPS-based predictive resource allocation in cellular networks was discussed in [5]. For location prediction, cell-to-cell transition probabilities of a mobile user are calculated according to previous inter-cell movements of the user. The transition probabilities are recorded in a transition matrix (TM). Based on this, resource allocation is done at the $\mathrm{k}$ most probable cells that are in the neighborhood of the current cell, where $\mathrm{k}$ is a user-defined parameter. Clustering mobile trajectories for resource allocation in mobile environments was introduced in [6]. This method is called Dynamic Clustering based Prediction (DCP) of mobile user movements which is used for the prediction of movements and dynamic allocation of resources depending on the collection of recorded mobile trajectories. Collected user trajectories are clustered according to their in-between similarity depending on weighted edit distance measures. The clustering used in [6] is agglomerative. It means that initially every single trajectory forms a cluster itself and at any iteration of the clustering algorithm, the two most similar clusters are merged to form a new cluster. Each new cluster is 
represented by a number of cluster representative trajectories. The merge operation continues until the number of clusters is reduced to a predefined value. A mobile user's next trajectory is predicted by finding the best matching representative with its current trajectory. The best matching one has the minimum edit distance to the current trajectory. In case of more than one match, all matched representatives can be used for prediction. Using such clustering technique will not match mobile network topology then Intra-cell and cell to cell prediction can't be achieved.

A data mining approach for location prediction in mobile environments is discussed in [7]. This method is used to predict the next cell of mobile user trajectory using sequential UMP algorithm without intra-cell prediction. So, it is only used to enhance network availability and aid in resource allocation with limited usage in accurate location based services and applications. The main difference between the two techniques presented in [6] and [7] is that; in [6], UAPs are clustered in order to mine the UMPs; while in [7] sequential pattern mining is used for the same purpose. Another difference is that only the next inter-cell movement of a mobile user is predicted in [7], while the complete trajectory of a mobile user is predicted in [6].

Another method for predicting future location of mobile user for location-based services system was discussed in [8]. This method is based on the movements of the user in the past. The prediction process in [8] consists of the following five steps:

1) Trajectories are reconstructed by re-sampling the moving points.

2) Trajectories are generalized on a graph; trajectory generalization plays a basic role in the data mining technique.

3) Historical movement database is transformed into a set of moving sequences prior to mining.

4) All frequent movement patterns are extracted using AllMOP algorithm.

5) Movement rules are extracted and users' next movements are predicted using RLP algorithm.

This method is based on GPS infrastructure. So, there is no deal with the mobile network topology which results in no enhancement in mobile network resource allocation and mobility management.

\section{Positioning in UMTS network platform}

UMTS is one of the third-generation ( $3 \mathrm{G})$ mobile telecommunications technologies. The Radio Access Network (RAN) is called UMTS Terrestrial RAN (UTRAN) and the radio access radio interface is also called UMTS Terrestrial Radio Access (UTRA). Variety of positioning methods has been specified for UTRAN networks such as:

\section{A- Cell -based method}

In this method, the target's position is detected corresponding to the coordinates of a nearby base station. Position data can be refined by taking into account the distance between the terminal and the base station, which can be derived from the RTT or the socalled Rx timing deviation, the angle of arrival (AoA) of signals from the terminal at the 
base station, or both of them. This method is reasonable for cell detection but it can't be used for map applications due to its low position accuracy as discussed in [9].

\section{B- Observed Time Difference Of Arrival with Idle Period Down Link (OTDOA-IPDL).}

This positioning method is basically the UTRAN counterpart of E-OTD and follows the same principles, that is, terminal-based method. However, timing measurements at the terminal suffer from the so-called hear ability problem, where the terminal might be unable to detect a sufficient number of neighbor base stations for OTD measurements and hence it is necessary to periodically interrupt downlink transmissions during the measurements. The control of IPDL is within Serving Mobile Location Centre (SMLC), which configures base stations for inserting the idle periods. The usage of IPDL results in solving downlink interruption in transmission during measurements.

\section{C- Uplink Time Difference Of Arrival (U-TDOA) in UTRAN}

U-TDOA is one of the three high-accuracy wireless location technologies now supported by the 3GPP. The technology, which has long since been standardized for AMPS, TDMA and CDMA systems is now being deployed throughout the U.S. to support phase II of the FCC's emergency 911 location mandate. U-TDOA technology locates wireless phones by comparing the time it takes a mobile station's radio signal to reach several Location Measurement Units (LMUs) installed at an operator's base stations, as shown in Figure 1. U-TDOA approach is used to enable location-based services such as emergency location, asset tracking, and mobile concierge services.

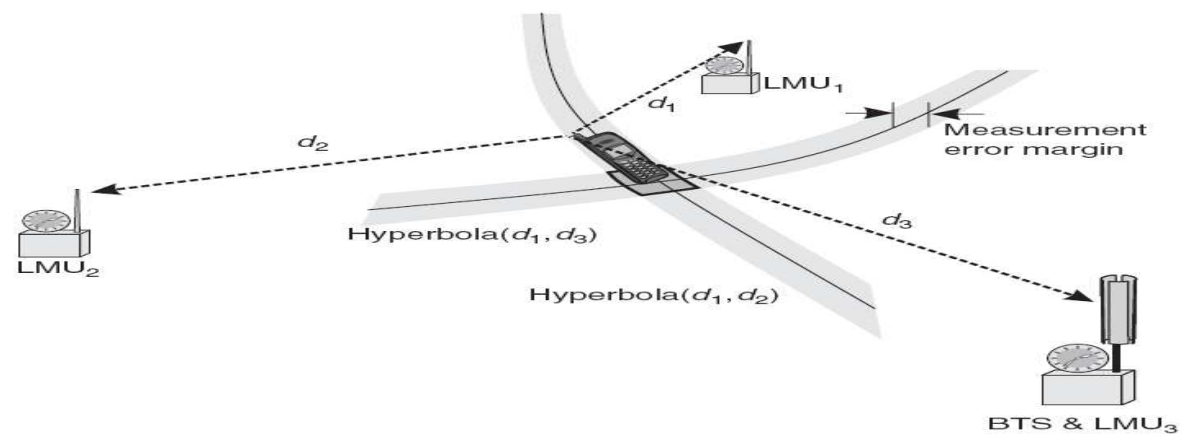

Figure (1) U-TDOA Network Configuration.

Uplink TDOA location method has proven highly accurate for location of wireless subscribers in CDMA based systems [9]. The wider UMTS bandwidth coupled with significant processing gain available to network based location systems provide higher level of accuracy in UTRAN networks and in situations where other location techniques may not be able to perform well. This increased accuracy can be achieved at reduced complexity over implementations of downlink OTDOA or A-GPS. Additionally, critical for future success of network operators, the uplink TDOA method provides significant flexibility for implementation of future location service enhancements. The U-TDOA location method performs well in urban, suburban, and indoor environments and less well in rural areas. 


\subsection{Advantages of Uplink TDOA in the UTRAN environment}

True position has spent over many years investigating potential location technologies and has concluded that uplink TDOA is the method that provides the best performance in the widest possible set of circumstances, at the lowest overall complexity and with the least impact on the wireless infrastructure [10]. In uplink method the processing functions to calculate user position is done in the network equipment especially location measurement unit (LMU) instead of mobile equipment processing used in downlink method. In addition, uplink method has increased processing capacity available to analyze signal information and to calculate subscriber locations. The uplink method provides increased power from 20 to $30 \mathrm{~dB}$ greater in processing gain than a DL-OTDOA solution through long integration times. In the DL-OTDOA system, the mobile station must make measurements of pilot signals from several sites, one by one, while still providing the other mobile station functions. The DSP processors of many LMUs work simultaneously to locate a single mobile subscriber. So, downlink method latency problem is solved. Simultaneous location of many subscriber units can be detected using U-TDOA approach utilizing increased power solution. Also, this solution includes acquisition of location information from many more distant location receivers.

Uplink TDOA provides protection against obsolescence. It is far easier and less complex to upgrade the software in RNCs and LMUs compared to upgrade millions of UEs needed in downlink methods. Uplink TDOA does not require implementation of the Idle Period Down-Link (IPDL) functionality, and it can operate in FDD and TDD networks. The Uplink TDOA solution, when integrated with the Node B will have a very low incremental cost.

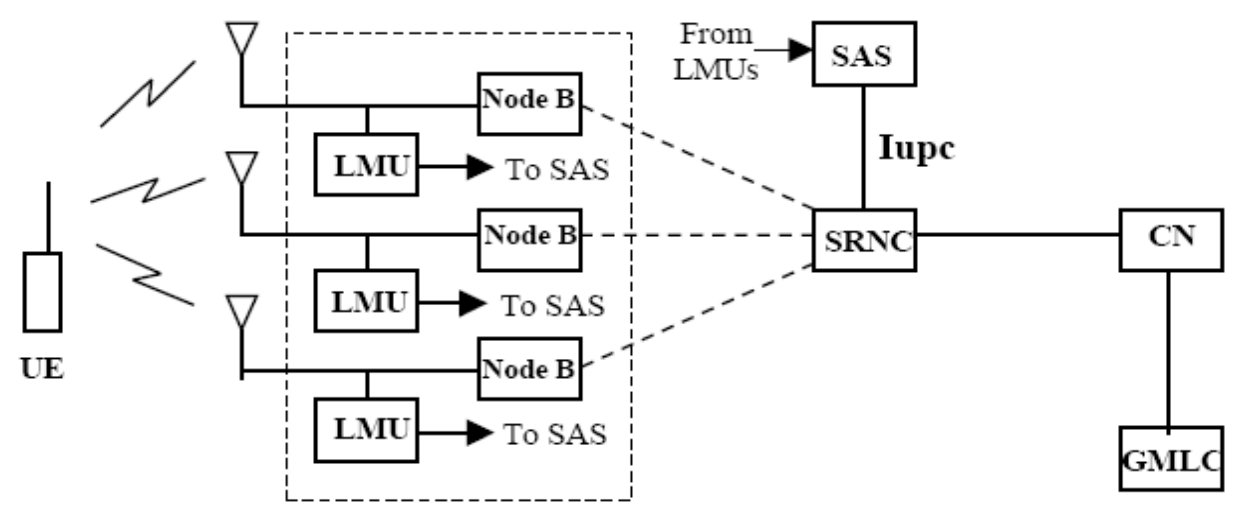

Figure (2) U-TDOA Network Structure Diagram.

\section{Usage of future location prediction in mobility management}

Location management has been significantly refined for the UMTS network architecture. Mobility management involves two processes as introduced in [11], location management and handoff management. Location management enables the 
wireless network to discover the current point of attachment of a mobile terminal and delivers calls to it. Whereas, handoff management enables the mobile network to locate roaming mobile terminals for call delivery and to maintain connection as the mobile terminal is moving around. LBSs can be supported by the operations of location management. During the first stage of location management, known as location registering or location update, the mobile terminal periodically informs the network by its new access point and helps the network to authenticate the user and revise the users' location profiles. The second stage is call delivery, in which the wireless mobile network is queried for a mobile terminal location and the current position of that terminal is found. On the other hand, handoff primarily represents a process of changing some of the parameters of a channel (frequency, time slot, spreading code, or a combination of these) while the current connection is in use. The handoff process usually consists of two phases: handoff initialization phase and handoff enabling phase. In the handoff initialization phase, the quality of the current communication channel is considered to decide when the handoff process should be triggered. In the handoff enabling phase, the allocation of new resources by new base station is initiated and processed. Poorly designed handoff schemes tend to generate huge signaling traffic, and thereby a dramatic decrease in quality of service (QOS) of integrated services in the wireless network.

\subsection{Location management strategies}

Location management strategies can be categorized into two main groups: static and dynamic. In static schemes, the way that users are paged is very much the same for all users and is determined in an off-line manner. Static scheme does not take into account any particular information about the users. While in dynamic schemes, different network topologies are used for different users. These topologies are highly related to the movement pattern and calling behavior of each user. It is obvious that dynamic schemes are much more complex than static ones and require more computation capabilities in the network [11].

\subsection{Basic location management strategies}

In this section the following seven basic location management strategies are reviewed.

(1) Always update strategy: In this scheme, a mobile terminal updates its location whenever it crosses a cell boundary. This enables the network to locate the user in minimum time for each incoming call. This means that the network will page the user only in the last updated cell. This scheme generates a massive number of unnecessary location update signals.

(2) Never update strategy: A mobile terminal never updates its location, and consequently, for each incoming call the network must page the user in all cells of the network. It is also obvious that this strategy is highly inefficient, especially when there are massive numbers of cells in the network. Naturally, as the number of users in the system increases, the number of simultaneous paging signals would also increase. 
(3) Time based strategy: In this method, a user updates its location after a predefined time span. In the case of an incoming call, the network will page the user in the last update cell and the possible cells that the user might be in after the last location update. Although this strategy seems better than the previous two, some disadvantages remain. For example, if the mobile terminal changes its location very frequently it becomes impossible to locate it in a timely fashion.

(4) Movement based strategy: The user performs a location update after passing a predefined number of cells; M. In this case, the network attempts to page the user in a radius of $\mathrm{M}$ cells starting from the last update cell. The main disadvantage of this strategy is in the case of the users who periodically crisscross (zigzag) adjacent cells of a network.

(5) Distance based strategy: In this case, the user updates its location after traveling for a predefined distance, $\mathrm{R}$, form the last update cell. The network can page the user in all cells that are physically within radius $\mathrm{R}$ of the last updated location. The main problem in this method is the need for knowledge of the topography of the network.

(6) Location Areas (LAs) scheme: This technique is adopted in the current GSM networks, where adjacent cells are grouped together to form a LA. In this case, the user performs an update whenever it leaves a LA and moves into a new one. Therefore, in the case of an incoming call, the network must page the user in all the cells of the last updated LA.

(7) Paging cells scheme: In this case, the user makes an update whenever it passes through predefined cells known as paging cells. A paging neighborhood is defined for each paging cell. The paging neighborhood of each paging cell contains all nonpaging cells of the network that must page the user in the case of an incoming call.

\subsection{Advanced location management strategies}

Traditional location management techniques for mobile networks are very conservative and there is a need for more 'intelligent' techniques' that endow networks with better location prediction capabilities. The usage of movement prediction patterns enhances the location update and paging processes. Also it reduces the execution time and the number of needed processes by divides users into two main categories; namely, a low mobility group and a high mobility group. For each we can use the suitable management strategy to overcome wasting resources or missing calls. Low and high mobility groups can be discovered smoothly using future location prediction method. The proposed algorithm presented in this paper is highly efficient in this respect as it is intra cell based method by which we can detect user movement scheme within a predefined time period. In other words future location of mobile user can be detected within time T. So, it will be simple for mobile network to manage paging process.

\section{Problem Definition}

In this article it is supposed that the mobile subscriber moves in UMTS mobile network. The covered area is divided into cells each with hexagonal shape and cells are divided into sectors. For example, assume that each cell contains three sectors as shown in Figure (3b). While user moves in mobile network from one cell to another, its movement will be recorded in a database named Home Location Register (HLR). Each 
base switching center has another database named Visitor Location Register (VLR). Each base station can detect the specific sector which serves the user. By applying one of the previously discussed methods for location detection we can detect the accurate position of the subscriber. The database structure is constructed from four fields [Pmn, $\mathrm{X}, \mathrm{Y}, \mathrm{T}]$ where, (Pmn) represents Cell ID (m) and sector ID (n), $(\mathrm{X}, \mathrm{Y})$ represent user coordinates (latitude and longitude respectively) and $\mathrm{T}$ is the time set of measure. The proposed method is based on three levels of location detection:

1- Intra-Sector Prediction (ISP) level: fully depends on user's accurate position within the specific sector and is used mainly to enhance LBS applications especially in urban and downtown areas.

2- Sector to Sector Prediction (SSP) level: no need for user coordinates. Only knowledge of user's cell and sector leads to establish this level of prediction which is used mainly to enhance intra-cell handover and network resources in addition to LBS applications.

3- Cell to Cell Prediction (CCP) level: using clustering technique as each $\mathrm{n}$ sectors represent one cell. Cell to cell prediction is highly effective in inter cell handover process beside LBSs applications especially in rural areas.

User trajectory is generally specified as Traj. $=\{$ Pmn, $\mathrm{x}, \mathrm{y}, \mathrm{ti}\}, \mathrm{i}=1: \mathrm{k}$; where $\mathrm{k}$ represents the trajectory's length. The determination of trajectory's length depends on the following assumptions:

1- If a mobile user stays at any point, cell or sector or subsector according to the used level of prediction, for more than a predefined threshold value before moving to another location then, we assume trajectory length ends at this point and any later movement will be considered as a start of another new trajectory. These trajectories are considered as Real Trajectories (RTJ) which looks like user actual path (UAP) presented in [7].

2- Moving Sequence (MS) is being defined as a list of temporally ordered cells, sectors or subsectors. A sequence maximum lifespan [start, end] where, start $\leq \mathrm{ti} \leq$ $\mathrm{tk} \leq \mathrm{end}$, for $\mathrm{k} \leq \mathrm{T}$ and $1 \leq \mathrm{i} \leq \mathrm{k}$.

3- Sequence composed of $k$ points is defined as k-pattern.

4- Subsequence generated if all points in sequence (S1) is contained in sequence (S2) with the same temporally direction flow then $\mathrm{S} 1$ is a subsequence of S2.

Frequent patterns can be extracted when real trajectory comply with moving sequence at a timestamp. Each moving sequence contains support value corresponding to number of trajectories in the cell, sector or subsector at a specific time set. If this value is equal to or greater than the predefined minimum support value (min_supp) then, the pattern is considered as frequent one pattern as specified in [8].

In this paper, the clustering technique used in [8] is adopted with limitations to meet the requirements of proposed method as will be discussed in the next section. Clustering method is suitable while predicting inter cell movement especially if intra cell prediction is useless.

Generated frequent patterns are used to produce prediction mobility rules. Full prediction process will be completed by using pre extracted mobility rules with a suitable confidence value. Figure 3, shows mobile network region distribution where each region is divided into cells, each cell is divided into three sectors. Each sector is 
labeled by cell id followed by a, b or $\mathrm{c}$ referring to the sector number 1,2 or 3 respectively.

For intra sector prediction (ISP) level, accurate coordinates (X, Y) are taken into consideration. Sub sector definition can be defined as a part of the sector in which user movements are discarded. The number and the size of the sub sector are defined based on the nature and capacity of the covered area in addition to the needed application.

A movement rule in this work is defined using the expression $\mathrm{A} \rightarrow \mathrm{B}$, where $\mathrm{A}, \mathrm{B}$ are moving sequences and $\mathrm{A} \cap \mathrm{B}=\varnothing$. The part of the rule before the arrow is the antecedent and the part after the arrow is the consequent of the rule. The confidence of a rule A B is known as an estimate of the probability $\mathrm{P}(\mathrm{B} \mid \mathrm{A})$. Namely, the probability of finding the consequent of the rule in moving sequences under the condition that these moving sequences also contain the antecedent A. Therefore, the confidence is defined as:

$$
\operatorname{Conf}(\mathrm{A} \rightarrow \mathrm{B})=\frac{\mathrm{SUP}(\mathrm{AU} \mathrm{B})}{\mathrm{SUP}(\mathrm{A})}
$$

In Equation (1), the support of the pattern (AUB) is the support of the rule and it is available after the step of frequent pattern discovery. However, the support of moving sequence $\mathrm{A}$ is not defined and we have to determine it. By selecting a suitable confidence value to achieve prediction, a decision for the next user's movement can be taken.

\section{Process of predicting user's future location}

In this work we proposed three levels of prediction, Sector to Sector Prediction (SSP), Intra-Sector Prediction (ISP) and Cell to Cell Prediction (CCP). ISP is internally contained in general SSP as regions which form ISP are parts of sectors in SSP method. The three levels of predictions are carried out using the following five main processes:

1. Process of map reconstruction on the organized network graph.

2. Moving sequences' creation process.

3. Extraction of all frequent movement patterns.

4. Generation of mobility rules.

5. Prediction of user's next movement.

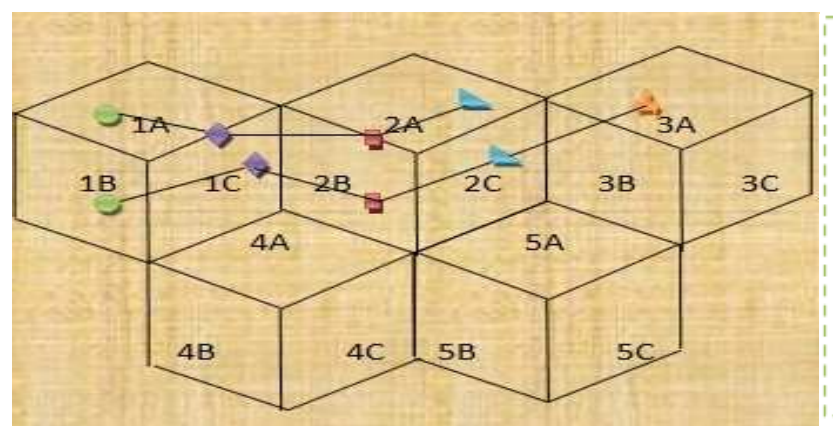

(a)

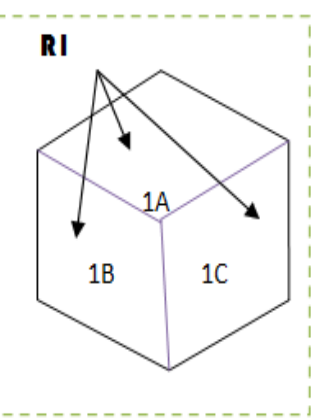

(b)

Figure (3) Distribution of Trajectories on Mobile Network Platform

\subsection{Map reconstruction.}

Our proposed map is formed by dividing the network graph into hexagonal cells and each cell is subdivided into sectors. The most common sectoring technique is to divide 
the cell into three sectors. Practically, each mobile operator can make its own actual division according to network topology and user capacity. By inserting the time factor of each trajectory, the proposed map is decomposed into spatiotemporal units. Spatiotemporal unit is defined as the minimum spatial and temporal extent of interest as discussed in [8].

\subsection{Finding moving sequences.}

Creation of moving sequences from corresponding input dataset is shown in figure (4) which requires definition of the following new parameters:

- (Ii): Sub-sector number, the area of the sector will be divided into sub areas to achieve intra-sector prediction level. The number of sub-sectors is determined according to the network topology, the surrounding environment and aimed accuracy of prediction. Studying intra-sector change requires no change in $\mathrm{m}, \mathrm{n}$ of the current point in comparison with the previous location. Significant change in $(\mathrm{Xi}-1, \mathrm{Xi})$ and $(\mathrm{Yi}-1, \mathrm{Yi})$ leads to change in sub-sector number.

- (Di): Intra-sector Enabling parameter, indicates change in (Ii) values at two consecutive timestamps within the same sector (i.e no change in $m, n$ value). This parameter used in auto-selective prediction type depending on change in intra-sector values (Ii-1,Ii). If $\mathrm{Di}=1$, then intra-sector change occurred else no intra-sector change occurred but cell to cell or sector to sector may occurred. This parameter is disabled in cell to cell and sector to sector prediction levels.

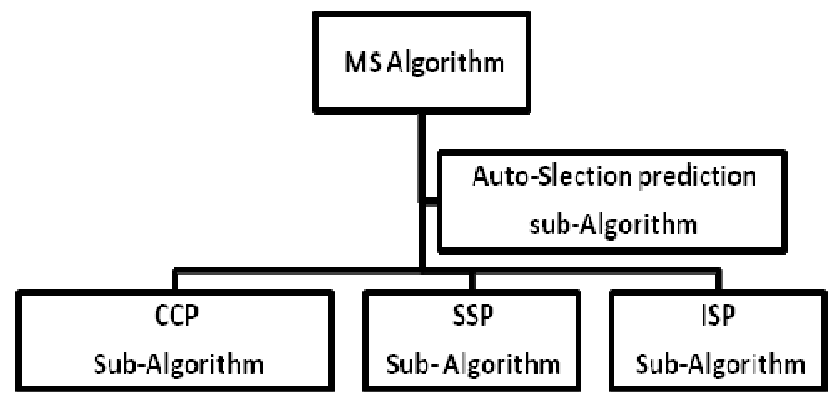

Figure (4) Creation chart of MS Algorithm with sub-categories components

Unfortunately cell sizes cannot be controlled as they are predefined by the network operator. Instead of controlling the cell size, time interval is controlled. User maximum velocity is the most important factor in choosing time period. Time intervals are chosen to be able to make at least one hit in each cell, sector or sub sector according to the adopted technique used in user trip. Thus, the following relation should be satisfied: $\mathrm{Vmax}^{*} \mathrm{t} \ll \mathrm{r}$; where $\mathrm{r}$ is the size of the region unit, $\mathrm{t}$ is the time difference from one point to another and Vmax is the user maximum velocity. In case of multiple hits per sector ISP is directly used to locate the accurate position of the user. If the same (Ii) points are repeated more than one time till $\mathrm{t}=\mathrm{Tmax}$, that means trajectory stopped there and any movement after that will be considered as a beginning of a new trajectory. The following algorithm is developed for MS creation including three different prediction types. 
The inputs of MS algorithm are:

- User's location point within timestamp, Location point: $(\mathrm{Pmn}, \mathrm{Ii}, \mathrm{Ti})$.

- User's real trajectory, Real trajectories (RTJ).

- Maximum length of points per trajectory, Max_span (Tmax).

The moving sequence creation process can be summarized in the following steps:

1) Initialize the set of moving sequence values; $M S \longleftarrow \phi$

2) Extract location of Mobile user in the form of (cell, sector, sub sector and Time set). Where, $(P i \longleftarrow(P m n, I i, T i))$.

3) Initialize the user's previous location; (Previous location (prev. loc.) $\leftarrow 1$ ).

4) Create moving sequence points according to three prediction types.

5) For (i=2; $i=$ max_span no. of points ; $i++)$

\section{5-A) Cell to Cell prediction (CCP)}

5-A-1) Disable sector and intra-sector prediction as user moves from one cell to another

$$
n_{i}, I_{i} \leftarrow \text { Disabled }
$$

5-A-2) Create moving sequence; if there is a change from one cell to another within two consecutive timestamps. Then, MS consists of previous MS in addition to the current obtained real trajectory that meets the previous conditions.

$$
\left.\begin{array}{c}
I f m_{i} \neq m_{i-1} \\
R T J=R T J \cup\left(P_{m i}, T_{i}\right) \\
M S=M S \cup R T J
\end{array}\right\}
$$

5-A-3) Stop moving sequence at the current cell; if the user still in the same cell from the current point $(\mathrm{Tc})$ till reaches Tmax. Any other movement after that is regarded as a start of new movement.

$$
\left.\begin{array}{c}
\text { If }\left(m_{i}=m_{i-1}\right),\left(T_{c} \leq t \leq T_{\max }\right) \\
M S \longleftarrow \text { Stop } \\
\operatorname{Re} t \text { turnMS }
\end{array}\right\}
$$

\section{5-B) Sector to Sector Prediction (SSP)}

5-B-1) Disable intra-sector prediction as user moves from one sector to another

$$
I_{i} \longleftarrow \text { Disabled }
$$

5-B-2) Create moving sequence; if there is a change from one sector to another within two consecutive timestamps. Then, MS consists of previous MS in addition to the current obtained real trajectory which meets previous conditions.

$$
\left.\begin{array}{c}
I f\left(P_{m n i} \neq P_{m n i-1}\right) \\
R T J=R T J \cup\left(P_{m n i}, T_{i}\right) \\
M S=M S \cup R T J
\end{array}\right\}
$$


5-B-3) Stop moving sequence at the current sector; if the user still in the same sector from the current point (Tc) till reaches Tmax. Then, any other movement after that is regarded as a start of new movement.

$$
\left.\begin{array}{c}
\text { If }\left(P_{m n i}=P_{m n i-1}\right),\left(T_{c} \leq t \leq T_{\max }\right) \\
M S \longleftarrow \text { Stop } \\
\operatorname{ReturnMS}
\end{array}\right\}
$$

\section{5-C) Intra-Sector prediction (ISP)}

5-C-1) Enable intra-sector number within prediction as user moves from sub sector to another.

$\left(I_{i} \leftarrow\right.$ Enabled $)$

5-C-2) Create moving sequence; if there is a change from one sub-sector to another within two consecutive timestamps. Then, MS consists of previous MS in addition to the current obtained real trajectory which meets previous conditions.

$$
\left.\begin{array}{c}
I f\left(P_{m n i}, I_{i}\right) \neq\left(P_{m n i-1}, I_{i-1}\right) \\
R T J=R T J \cup\left(P_{m n i}, I_{i}, T_{i}\right) \\
M S=M S \cup R T J
\end{array}\right\}
$$

5-C-3) Stop moving sequence at the current sub-sector; if the user located in the same sub-sector of the same cell and sector from the current point (Tc) till reaches Tmax. Any other movement after that is regarded as a start of new movement.

$$
\left.\begin{array}{c}
\text { If }\left(\left(P_{m n i}, I_{i}\right)=\left(P_{m n i-1}, I_{i-1}\right)\right),\left(T_{c} \leq t \leq T_{\max }\right) \\
M S \longleftarrow \text { Stop } \\
\operatorname{Re} t u r n M S
\end{array}\right\}
$$

Entra-sector enabling (Di) parameter enables auto-selection of the used MS patterns instead of manual choice. The hereunder algorithm shows auto-selection process:

\section{5-D) Auto-Selection of MS pattern}

5-D-1) Enable Sub-sector prediction parameter (Di) which indicate user's movement level

$$
\text { If }\left(D_{i}=1\right)
$$

5-D-2) change in sub-sector prediction level; intra sector changes within the same main cell and sector occurred, So ISP level is used.

$$
\left.\begin{array}{c}
\operatorname{Goto}(5-C) \rightarrow " I S P " \\
\text { ElseIf }\left(D_{i}=0\right)
\end{array}\right\}
$$

5-D-3) Check sector to sector changes; if there is movement from one sector to another within the same main cell. Then, SSP prediction is used.

$$
\left.\begin{array}{c}
\text { If }\left(n_{i} \neq n_{i-1}\right), \text { While }\left(m_{i}=m_{i-1}\right) \\
\operatorname{Goto}(5-B) \rightarrow " S S P^{\prime \prime}
\end{array}\right\}
$$


Else, cell to cell prediction is the proper MS algorithm

$$
\text { Else, Goto }(5-A) \rightarrow " C C P "
$$

\subsection{Finding movement frequent patterns.}

As ALLMOP algorithm is based on generalized sequential pattern (GSP) algorithm with additional temporal join operation and different method for pruning candidates. The application of clustering technique to control dense regions is introduced in [8]. The proposed algorithm of Intra-cell Movement Pattern (ICMP) benefits from ALLMOP algorithm but with suitable modifications to be matched with intra-cell prediction requirements based on mobile network platform. To fully utilize mobile network structure and environment nature (i.e. urban, rural, downtown, highway, etc) ICMP algorithm will be divided into sub algorithms to cover all prediction requirements in mobile network as shown in figure 5. In some rural area intra-cell prediction may not be necessary due to low density of mobile subscribers. So, cell to cell prediction is preferred to save network resources instead of intra cell prediction. Here, clustering technique is adopted but with some limitations as it is not accepted to make random clustering for any neighboring cells as proposed in [8]. Cluster in this proposed work can be defined as a collection of three neighboring sectors which form the cell of the network (e.g. sectors 1A,1B,1C in Figure 3 are collected to form cluster R1). Cluster's selection limitation yields to save network topology and support directly in cell to cell movements predictions.

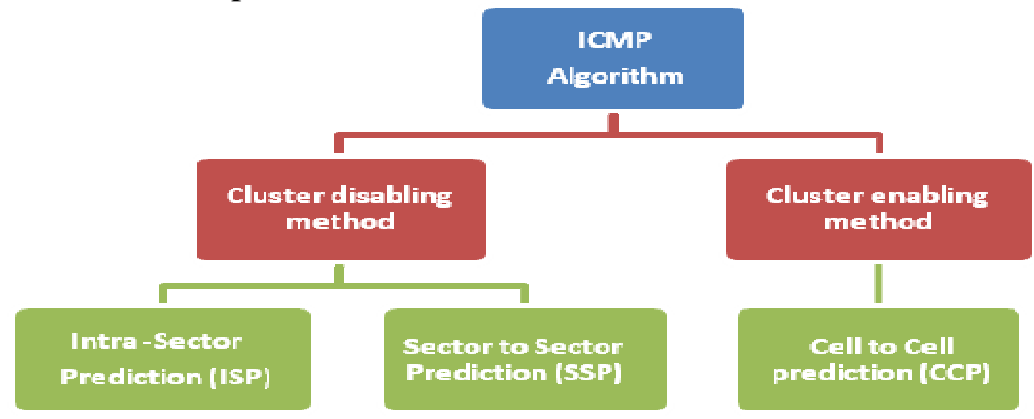

Figure (5) ICMP Algorithm Main Components.

In urban regions and downtime environment, intra cell prediction is useful comparable with the used resources for accurate location detection. Here, clustering technique can't be used. The usage of intra cell prediction depends on the required accuracy of location prediction application.

\subsubsection{Generating frequent- 1 pattern.}

As shown in Table 1, a dataset of real trajectories are decomposed into groups of corresponding moving sequences. Then, frequent- 1 patterns represent dense sectors or clusters that contain at least the minimum support points at specific timestamp.

\section{- ICMP cluster enabling algorithm.}

In this algorithm each three sectors within the same cell will be considered as one cluster. For example, sectors 1A, 1B, 1C are considered as cluster R1 as shown in 
Figure (3a). Assume that minimum support value is 2 (min_Sup $\geq 2$ ) then R1 will be considered as a frequent one pattern at timestamp T1 where R1 contains two points in time T1. By the same way we can produce frequent-1 pattern depending on clustering technique as shown in Table 2.

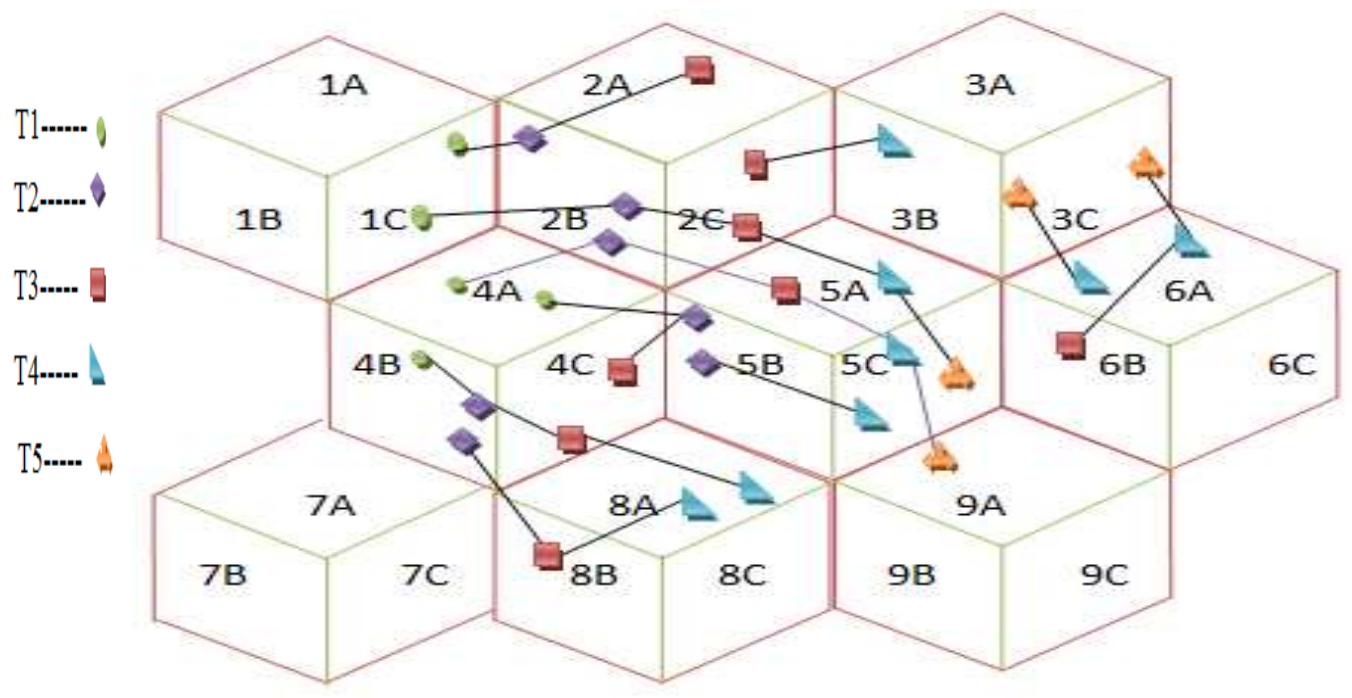

Figure (6) Full Trajectories on A Map Based on UMTS Network Graph.

Table (1) Finding Moving Sequences of Real Trajectories

\begin{tabular}{|c|c|}
\hline $\begin{array}{c}\text { Real trajectory No. } \\
\text { [RTJ(i)] }\end{array}$ & $\begin{array}{c}\text { Moving Sequence } \\
{[\text { Pmn, T, D] }}\end{array}$ \\
\hline 1 & $\langle(1 \mathrm{C}, \mathrm{t} 1,0),(2 \mathrm{~B}, \mathrm{t} 2,0),(2 \mathrm{~A}, \mathrm{t} 3,0)\rangle$ \\
\hline 2 & $\langle(1 \mathrm{C}, \mathrm{t} 1,0),(2 \mathrm{~B}, \mathrm{t} 2,0),(2 \mathrm{C}, \mathrm{t} 3,0),(5 \mathrm{~A}, \mathrm{t} 4,0),(5 \mathrm{C}, \mathrm{t} 5,0)\rangle$ \\
\hline 3 & $\langle(2 \mathrm{C}, \mathrm{t} 3,0),(3 \mathrm{~B}, \mathrm{t} 4,0)\rangle$ \\
\hline 4 & $\langle(4 \mathrm{~A}, \mathrm{t} 1,0),(2 \mathrm{~B}, \mathrm{t} 2,0),(5 \mathrm{~A}, \mathrm{t} 3,0),(5 \mathrm{C}, \mathrm{t} 4,0),(9 \mathrm{~A}, \mathrm{t} 5,0)\rangle$ \\
\hline 5 & $\langle(4 \mathrm{~B}, \mathrm{t} 1,0),(4 \mathrm{~B}, \mathrm{t} 2,1),(4 \mathrm{C}, \mathrm{t} 3,0),(8 \mathrm{~A}, \mathrm{t} 4,0)\rangle$ \\
\hline 6 & $\langle(4 \mathrm{~A}, \mathrm{t} 1,0),(5 \mathrm{~B}, \mathrm{t} 2,0),(4 \mathrm{C}, \mathrm{t} 3,0)\rangle$ \\
\hline 7 & $\langle(5 \mathrm{~B}, \mathrm{t} 2,0),(5 \mathrm{C}, \mathrm{t} 3,0)\rangle$ \\
\hline 8 & $\langle(6 \mathrm{~B}, \mathrm{t} 3,0),(6 \mathrm{~A}, \mathrm{t} 4,0),(3 \mathrm{C}, \mathrm{t} 5,0)\rangle$ \\
\hline 9 & $\langle(6 \mathrm{~A}, \mathrm{t} 4,0),(3 \mathrm{C}, \mathrm{t}, \mathrm{s}, 0)\rangle$ \\
\hline 10 & $\langle(4 \mathrm{~B}, \mathrm{t} 2,0),(8 \mathrm{~B}, \mathrm{t} 3,0),(8 \mathrm{~A}, \mathrm{t} 4,0)\rangle$ \\
\hline
\end{tabular}

The cells which contain less than minimum support number of points within specific timestamp will be considered as outliers as $\langle\mathrm{R} 6, \mathrm{t} 3\rangle$ and $\langle\mathrm{R} 9, \mathrm{t} 5\rangle$. Empty cells will be discarded as cell R7.

\section{- ICMP cluster disabling algorithm.}

In this algorithm, movement is based on sector or intra sector prediction level without using clusters.

Each sector or intra sector contains the minimum support number of points at specific timestamp will be regarded as frequent -1 pattern. While using SSP method, if 
the same trajectory hits the sector more than one time before it moves to the next sector only the first hit is taken into consideration and other hits are neglected. If the user still in the same sector till Tend, that means trajectory movement is terminated at this point, only the first movement will be taken into consideration.(e.g. $\langle(4 \mathrm{~B}, \mathrm{t} 2)\rangle$ will be discarded and moving sequence of this trajectory will be $[\langle(4 \mathrm{~B}, \mathrm{t} 1)\rangle,\langle(4 \mathrm{C}, \mathrm{t} 3)\rangle,\langle(8 \mathrm{~A}, \mathrm{t} 4)\rangle])$. According to this procedure, $\langle(4 \mathrm{~B}, \mathrm{t} 2\rangle$ is not frequent pattern as it contains one support point.

In ISP method, intra sector movement is taken into consideration if $\mathrm{Di}=1$, (i.e. $(\mathrm{Xi}, \mathrm{Yi})$ of the current movement is different by sufficient predefined value from the previous obtained value) then [ (Ii) $\neq($ Ii-1)]. At this method, U_TDOA method is used to make accurate positioning. The moving sequence is created on the form of [Pmn, I, $\mathrm{T}, \mathrm{D}]$. so, The moving sequence of RTJ(5) is defined as $<(4 \mathrm{~B}, 1, \mathrm{t} 1,0),(4 \mathrm{~B}, 2, \mathrm{t} 2,1)$, $(4 \mathrm{C}, 1, \mathrm{t} 3,0),(8 \mathrm{~A}, 1, \mathrm{t} 4,0)\rangle$. In this pattern we can observe that $\langle(4 \mathrm{~B}, \mathrm{t} 2)\rangle$ is a frequent- 1 pattern as it matches the minimum support points.

Table (2) Example of Extracting Frequent-1 Pattern in Cluster Enabled Algorithm

\begin{tabular}{|c|c|}
\hline $\begin{array}{c}\text { Frequent-1 pattern " Cluster } \\
\text { enabled method" }\end{array}$ & Support value \\
\hline$<(\mathrm{R} 1, \mathrm{t} 1)>$ & 2 \\
\hline$<(\mathrm{R} 2, \mathrm{t} 2)>$ & 3 \\
\hline$<(\mathrm{R} 2, \mathrm{t} 3)>$ & 3 \\
\hline$<(\mathrm{R} 3, \mathrm{t} 5)>$ & 2 \\
\hline$<(\mathrm{R} 4, \mathrm{t} 1)>$ & 3 \\
\hline$<(\mathrm{R} 4, \mathrm{t} 2)>$ & 2 \\
\hline$<(\mathrm{R} 4, \mathrm{t} 3)>$ & 2 \\
\hline$<(\mathrm{R} 5, \mathrm{t} 2)>$ & 2 \\
\hline$<(\mathrm{R} 5, \mathrm{t} 3)>$ & 2 \\
\hline$<(\mathrm{R} 5, \mathrm{t} 4)>$ & 2 \\
\hline$<(\mathrm{R} 6, \mathrm{t} 4)>$ & 2 \\
\hline$<(\mathrm{R} 8, \mathrm{t} 4)>$ & 2 \\
\hline
\end{tabular}

\subsubsection{Generating frequent-k patterns}

Frequent- 2 pattern is created by joining frequent- 1 pattern with itself. This definition is suitable for any of ICMP sub algorithms each with its related frequent-1 pattern. Movement direction and time order should be taken into consideration where, transition from cell 1 to cell 2 is different from movement from cell 2 to cell 1 . Movement from one cell to another should take into consideration that both cells are neighbors and comply with the real trajectory.

Frequent-k pattern, where $\mathrm{K}>2$ can be extracted from joining frequent-k-1 pattern with frequent-1 pattern with condition that, the two patterns related to the same trajectory and achieve minimum support value. 
The following algorithm is developed for ICMP-algorithm creation with clustering enabled and disabled sub-patterns. The inputs of the ICMP algorithm are:

- Moving sequence (MS)

- Minimum support (min_sup)

- Prediction patterns (CCP, SSP, ISP)

The ICMP-algorithm creation process can be summarized into the following steps:

1- Initialize the frequent movement-1 pattern with Maximal timestamp among all movement of MS. $(F 1 \leftarrow \phi)$

2- For each ti , $\left(i=1, i \leq T, i_{++}\right)$

\section{2-A) For clustering enabled sub-pattern:}

2-A-1)_Merge each three sectors related to same cell into one cell, $X$ represent cell ID $\operatorname{Cell}(X) \leftarrow \operatorname{Sectors}(X A \cup X B \cup X C) \quad, \mathrm{A}, \mathrm{B}, \mathrm{C}$ are sectors of cell $(\mathrm{X})$

2-A-2) Define Region (R) as a Cell (x) with minimum number of points at timestamp (ti) and discarding outliers.

$$
\left.\operatorname{Re} \operatorname{gion}(R) \leftarrow\left(\operatorname{Cell}(X), T_{i}\right) \geq \text { min_sup }\right)
$$

2-A-3) Discard intra-sector prediction.

$$
D_{i} \leftarrow \text { Disabled }
$$

2-A-4) Extract (frequent-1 pattern) within specific time (ti).

$$
F 1 \leftarrow F 1 \cup\left(R, t_{i}\right)
$$

2-A-5) Initialize (Candidate-2) and (Frequent-2) patterns.

$$
(C 2 \leftarrow \phi, F 2 \leftarrow \phi)
$$

2-A-6) Generate (frequent-2) patterns, by combining two neighbor freq-1 patterns, with reasonable time order and with minimum support value of moving sequences.

$$
\begin{aligned}
& \text { Then, } \forall F 1\left(R_{i}, T i\right) \in F 1 \\
& \text { If }\left(T_{i}>T_{j}\right), R_{i}, R_{j} \text { neighbor_cells }, i \neq j \\
& C 2 \leftarrow C 2 \cup<\left(R_{i}, T_{i}\right),\left(R_{j}, T_{j}\right)> \\
& \text { If }\left(M S\left[R_{i} \rightarrow R_{j}\right] \geq \min \_\right. \text {Sup } \\
& \text { Then, } F 2=F 2 \cup<\left(R_{i}, T_{i}\right),\left(R_{j}, T_{j}\right)>
\end{aligned}
$$

2-A-7) Generate infrequent list including non-frequent patterns to discard them in freq k-patterns as implemented in [8].

$$
\text { Else, insert(Infre_list, Cand) }
$$

2-A-8) Generate (frequent k-pattern), $k>2$, is defined by combining frequent ( $k-1)$ with frequent-1 pattern with reasonable time order and with minimum support value of moving sequences otherwise, update of infrequent list is occurred as shown in [8]. 


$$
\begin{gathered}
\operatorname{For}\left(F_{k-1}\right) \neq \phi \\
\forall\left(P_{i}\right) \in F_{k-1} \\
\forall(P j) \in F-1 \\
\text { Cand }-k \leftarrow \text { joining }\left(P_{i}, P_{j}\right) \in M S \\
\text { If }(\text { Cand }-K) \neq \phi \\
\text { Then, } C k \leftarrow C k \cup \text { Cand }-k \\
\text { If }\left(M S\left[P_{K-1} \rightarrow P_{j}\right] \geq\right. \text { min_sup } \\
F_{k} \leftarrow\left(F_{k-1} \cup F 1\right)
\end{gathered}
$$

Else, update (Infreq_list, candK)

2-A-9) Create all Frequent patterns in cluster enabled method.

$I C M P$ _Clistering - enable $\leftarrow$ Set of all frequent sequences in $\mathrm{F}_{\mathrm{k}}$.

\section{2-B) Clustering disabled sub-pattern "Sector to sector prediction"[SSP]}

2-B-1) Represent [sector to sector prediction] as a type of clustering disabled technique.

Regions consist of sectors with min. support points at timestamp after discarding outliers.

$$
\operatorname{Re} \operatorname{gion}(R) \leftarrow\left(P_{m n}\right)
$$

2-B-2) Discard intra sector change. Then, same procedure is applied from equation 16 to 19 to get all possible frequent patterns.

$$
\left.\begin{array}{l}
\text { Di } \leftarrow \text { Disabled } \\
\text { Goto } 16 \rightarrow 19
\end{array}\right\}
$$

$I C M P-C l u s t e r i n g$ _Disabled $(S S P) \leftarrow$ Set of all frequent sequences in $\mathrm{F}_{\mathrm{k}}$.

\section{2-C) Clustering disabled sub-pattern "Intra-Sector prediction"[ISP]}

2-C-1) Represent [Intra-sector prediction] as a type of clustering disabled technique.

Regions consist of intra sectors with min. support points at timestamp after discarding outliers.

$$
\operatorname{Re} \operatorname{gion}(R) \rightarrow\left(P_{m n}, I\right)
$$

2-C-2) Enable intra sector change. Then, same procedure is applied (from equation16 to 19) to get all possible frequent patterns.

$$
\begin{gathered}
\text { Di } \leftarrow \text { Enabled } \\
\quad \text { Goto } 16 \rightarrow 19
\end{gathered}
$$

ICMP - Clustering _ Disabled $(S S P) \leftarrow$ Set of all frequent sequences in $\mathrm{F}_{\mathrm{k}}$. 


\section{Finding movement rules and next movement prediction.}

Movement rules are generated in this section depending on frequent movement patterns which are obtained previously. Independent of the ICMP sub pattern used, the pre obtained movement patterns are temporal joint points. Dealing with movement pattern $[C(R+, 1),(R 2, t 2),(R 5, t 3)>]$, this pattern is obtained from cluster enabling algorithm. This pattern represent frequent-3 pattern and has the possible movement

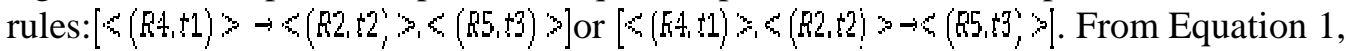
the confidence value of the two possibilities should be evaluated. The confidence threshold value is submitted according to the required prediction accuracy. The rules of the pattern that met the threshold confidence value are selected and other rules are discarded. The first assumption in the previous example has a confidence $=$ [ Sup ( $(\mathrm{R} 4, \mathrm{t} 1) \mathrm{U}(\mathrm{R} 2, \mathrm{t} 2) \mathrm{U}(\mathrm{R} 5, \mathrm{t} 3))$ / $\mathrm{Sup}(\mathrm{R} 4, \mathrm{t} 1)]$. As there is only one trajectory met the movement pattern $<(\mathrm{R} 4, \mathrm{t} 1),(\mathrm{R} 2, \mathrm{t} 2),(\mathrm{R} 5, \mathrm{t} 3)>$ then, $\operatorname{Sup}((\mathrm{R} 4, \mathrm{t} 1) \mathrm{U}(\mathrm{R} 2, \mathrm{t} 2) \mathrm{U}(\mathrm{R} 5, \mathrm{t} 3))=1$. Whereas, Sup $(\mathrm{R} 4, \mathrm{t} 1)=3$ points. So, confidence of the first assumption $=1 / 3=$ $33.33 \%$. The second assumption has a confidence $=[\operatorname{Sup}((\mathrm{R} 4, \mathrm{t} 1) \mathrm{U}(\mathrm{R} 2, \mathrm{t} 2) \mathrm{U}(\mathrm{R} 5, \mathrm{t} 3)) /$ $\operatorname{Sup}((\mathrm{R} 4, \mathrm{t} 1) \mathrm{U}(\mathrm{R} 2, \mathrm{t} 2))$ ]. As only one trajectory met the movement pattern <( $(\mathrm{R} 4, \mathrm{t} 1),(\mathrm{R} 2, \mathrm{t} 2)>$ then, $\operatorname{Sup}((\mathrm{R} 4, \mathrm{t} 1) \mathrm{U}(\mathrm{R} 2, \mathrm{t} 2)=1$. The confidence of this assumption $=$ $1 / 1=100 \%$. If the min_confidence value is selected to be $100 \%$ then, the movement rule $[C(R+1) \times(R 2,2] x \rightarrow C(R 5,+3)>]$ is taken into consideration. The second movement rule $[\zeta(A,+1)>\langle<(R 2, t 2)><(B 5,+3)>]$ is discarded. By applying these movement rules on frequent-k patterns, next movement of mobile user will be predicted according to predefined confidence.

Recursive depth fashion will be used to improve the procedure of movement rules as

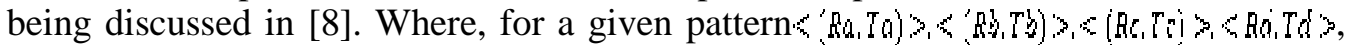

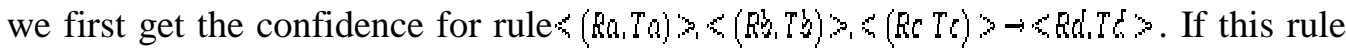

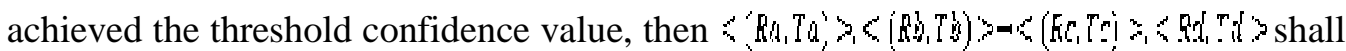
be studied. If that pattern doesn't achieve the confidence threshold, there is no need to check sub patterns of this pattern as it surely not achieve the confidence value too and that is matched with the previously obtained results.

ICMP rules for location prediction can be clarified within the following algorithm.

The inputs of the ICMP rules are:

- Minimum Confidence (Min_conf.)

- Frequent movement pattern (K-pattern) , $\mathrm{K} \geq 1$

The algorithm used in rules creation can be summarized in the following steps:

1- Initialize the value of the prediction rule

$$
\text { Rule }=\phi
$$

2- Calculate support value of k-pattern and k-1 pattern

$$
\begin{gathered}
P_{k} \in K-\text { pattern } \\
\operatorname{Sup}\left(P_{k}\right) \leftarrow A \\
P_{y} \in K-1 \text { pattern } \\
\operatorname{Sup}\left(P_{k}\right) \leftarrow B
\end{gathered}
$$


3- Calculate the confidence value of the first generated rule.

$$
\operatorname{Conf}(P)=\frac{\operatorname{Sup}\left(P_{k}\right)}{\operatorname{Sup}\left(P_{y}\right)}=\frac{A}{B}
$$

4- Compare the calculated confidence value with minimum confidence value adjusted to meet the required accuracy of prediction. If calculated pattern confidence value is greater than the predefined minimum confidence value then, the pattern is considered movement rule otherwise, it is not a prediction rule.

$$
\begin{aligned}
& \text { Min_Conf } \longleftarrow \text { Minimum Confidence } \\
& \text { If }(\operatorname{Conf}(P)) \geq \text { Min_Conf } \\
& \left.\begin{array}{c}
\text { Then, Rule }=\text { Rule } \cup<\left(P_{y}\right) \rightarrow\left(P_{k-y}\right)> \\
\text { Else, Return rule }
\end{array}\right\}
\end{aligned}
$$

5- Apply recursive depth fashion in finding rules of movements as applied in [8].

For $\mathrm{P}(\mathrm{y}-\mathrm{i}), \quad(1<\mathrm{i}<\mathrm{y}-1)$, where $\mathrm{P}(\mathrm{y}-\mathrm{i})$ is pattern $\mathrm{y}$ after dropping the last $\mathrm{i}$ th location.

$$
\left.\begin{array}{c}
\operatorname{Conf}\left(P_{i}\right)=\frac{\operatorname{Sup}\left(P_{k}\right)}{\operatorname{Sup}\left(P_{y-1}\right)} \\
\text { If }\left(\operatorname{Conf}\left(P_{i}\right) \geq \operatorname{Min} \_\right. \text {Conf } \\
\text { Then, Rule }=\text { Rule } \cup<P(y-i) \rightarrow P(k-y+i)> \\
\text { Else, return rule. }
\end{array}\right\}
$$

\section{Experimental Results}

The experimental results are obtained by performing the synthetic datasets and estimating the performance of our algorithm. Results are adopted according to simulation model. The adopted model is general and flexible to be matched with variety of understudy cases. Experiments are executed on C\# basis with a $2.39 \mathrm{GHZ}$ Intel(R) core(TM) 2 Due CPU, 1.95 GB of RAM running on Windows XP operating system.

\subsection{Dataset generation}

To simulate the movement of the mobile user, random data generator based on the used adopted algorithm is developed. Selection of neighbor cells represents the main difference between the three proposed algorithms. In cell to cell algorithm, trajectory points are distributed on adjacent cells. Whereas, these points are distributed on adjacent sectors or intra-sector regions while using sector to sector and intra-sector algorithms respectively. Number of points used to define trajectory length is selected to vary from minimum (Lmin) to maximum (Lmax) value depending on the required application. Total number of trajectories per user (TR) is adopted. By using mxn cells where $\mathrm{m}, \mathrm{n}$ represent the map size length and width respectively. Table (3) includes the used parameters in generating and processing data with default values used in this approach. Using default values results in generating 1000 trajectories distributed on $4 \times 3$ cells. Each trajectory consists of three points at least and seven points at most as a default values. 
Generated data also follow the following assumptions: on average $70 \%$ of user movements have the maximal lengths (Lmax) from which average $40 \%$ of those trajectories follow the same trip. So, 30\% of user movements are regarded as arbitrary trajectories. The proposed simulator is designed to enable user to adjust the input parameters. This leads system to be more flexible while applying variety of working conditions.

\subsection{Daily basis parameters}

The adopted algorithm deals with input data on daily basis, by selecting the number of trajectories which are included daily within specific timestamps. Daily input trajectories introduced can be equally or arbitrary according to the required application and user life style. In this approach the default value of trajectories per day (TRday) are 100 trajectories on equal daily basis.

In large number of input trajectories, system capacity (SC) parameter is introduced to limit the number of trajectories used to evaluate prediction rules at a specific time set. Decreasing system capacity will improve the execution time but it will decrease the precision of the obtained predictions.

Table (3) Summary of parameters

\begin{tabular}{|c|c|c|}
\hline Symbol & Definition & Default values \\
\hline TR & Total Number of trajectories per user in the proposed map & 1000 \\
\hline Lmin & Minimum number of points per trajectory & 3 \\
\hline Lmax & Maximum number of points per trajectory & 7 \\
\hline TRday & Number of estimated trajectories per day & 100 \\
\hline M & Map size = (mxn) cells , m=length , n=width & $4 \times 3$ \\
\hline SC & $\begin{array}{c}\text { System capacity : No. of last trajectories used to evaluate } \\
\text { predictions }\end{array}$ & 1000 \\
\hline Min_Sup & Minimum support value & 2 \\
\hline Min conf & Percentage of Minimum Confidence & 80 \\
\hline
\end{tabular}

\subsection{Evaluation of Precision and recall}

Precision and recall evaluation indicate system performance and prediction algorithm accuracy.

- Recall: the number of correctly predicted cells divided by the total number of requests Thus, the recall counts the "no-prediction" case as an incorrect prediction. This metric can be used to answer the question "How many relevant locations are returned?" High recall implies few false negatives.

- Precision: the number of correctly predicted cells divided by the total number of predictions made. This metric is appropriate for applications that may prefer no prediction to a wild guess. Precision is used to answer the question: "How many of the returned locations predicted are relevant?" High precision refers to few false positives [8]. 

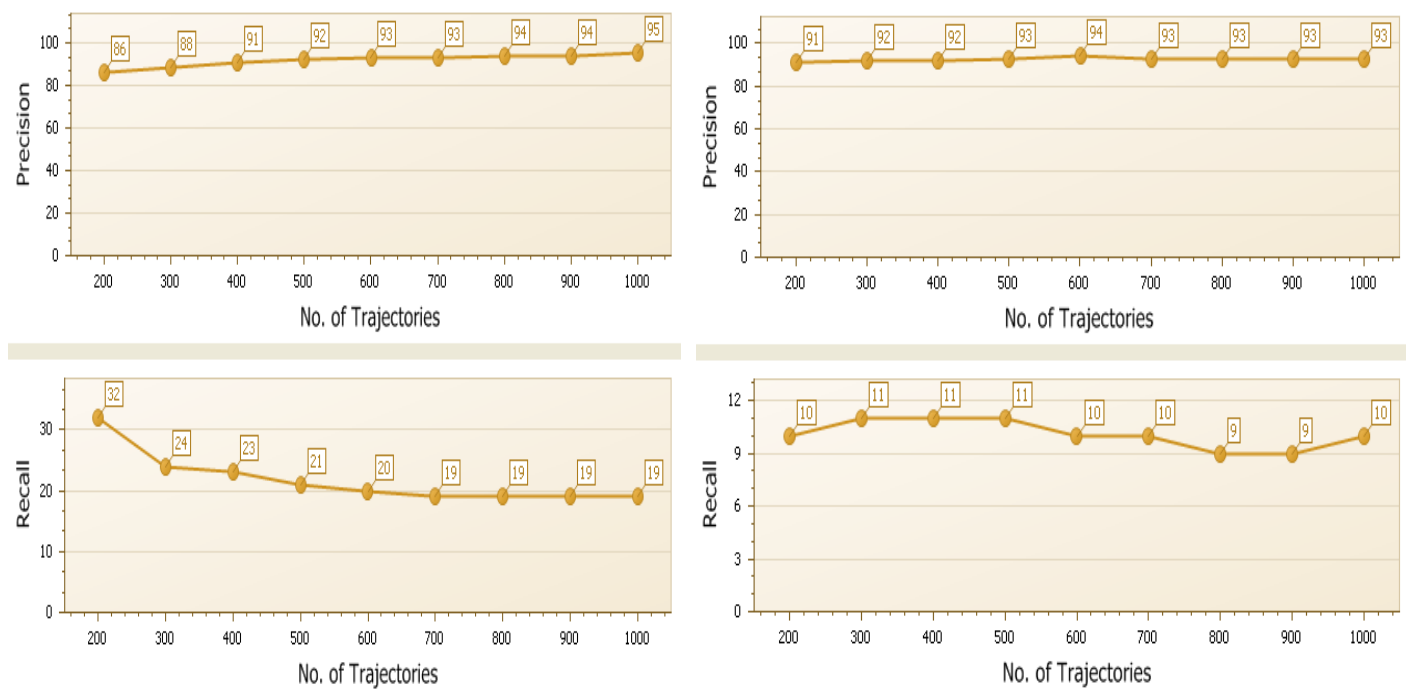

(a)

(b)
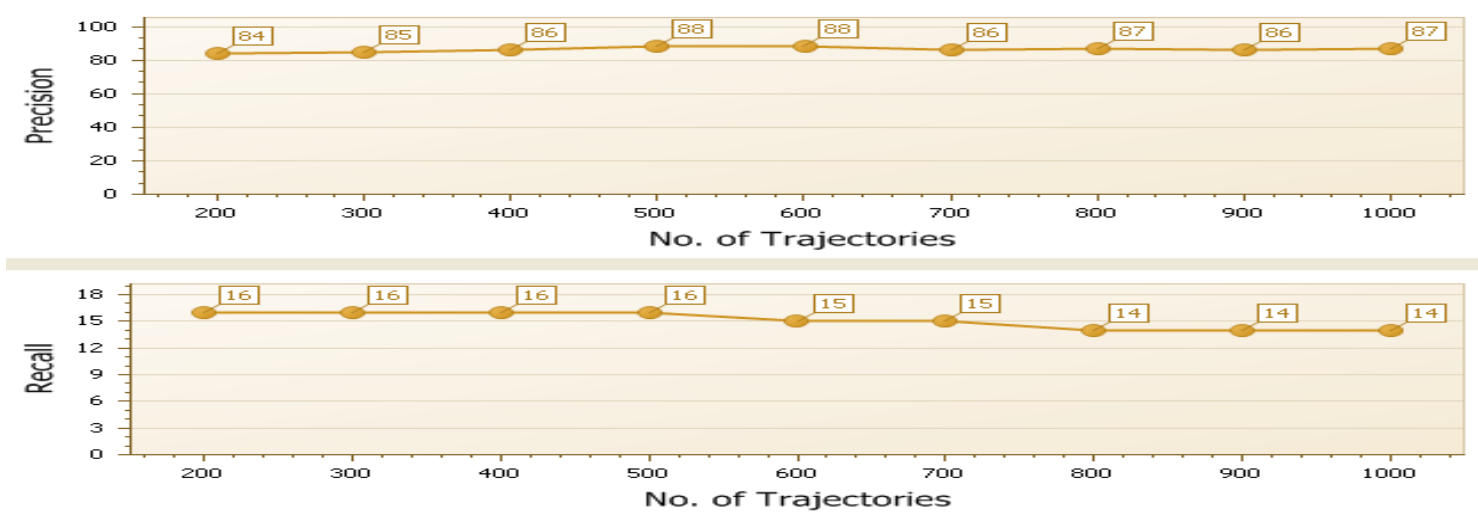

(c)

Figure (7) (a) Precision and Recall VS Trajectories Number Using Cell To Cell Algorithm. (b) Precision and Recall VS Trajectories Number Using Sector To Sector Algorithm. (c) Precision and Recall VS Trajectories Number Using ISP Algorithm

Using cell to cell, sector to sector and intra-sector algorithms with 1000 trajectories in $4 \times 3$ map, minimum support $=2$, minimum confidence $=80$ and 100 trajectories per day, system precision and recall is estimated as shown in figure (7).

\subsection{Effect of Minimum support}

Here, we study the impact of minimum support (Min sup) values on the precision and recall percentage of proposed algorithms. Figure (8) shows the decrement of precision and recall values by increasing min sup value. 
By increasing the min sup value, the number of generated rules will be decreased then the number of correct predictions will be decreased and results in decrease in both precision and recall percentages so, the optimum min sup value in the mentioned case is the default value $=2$.

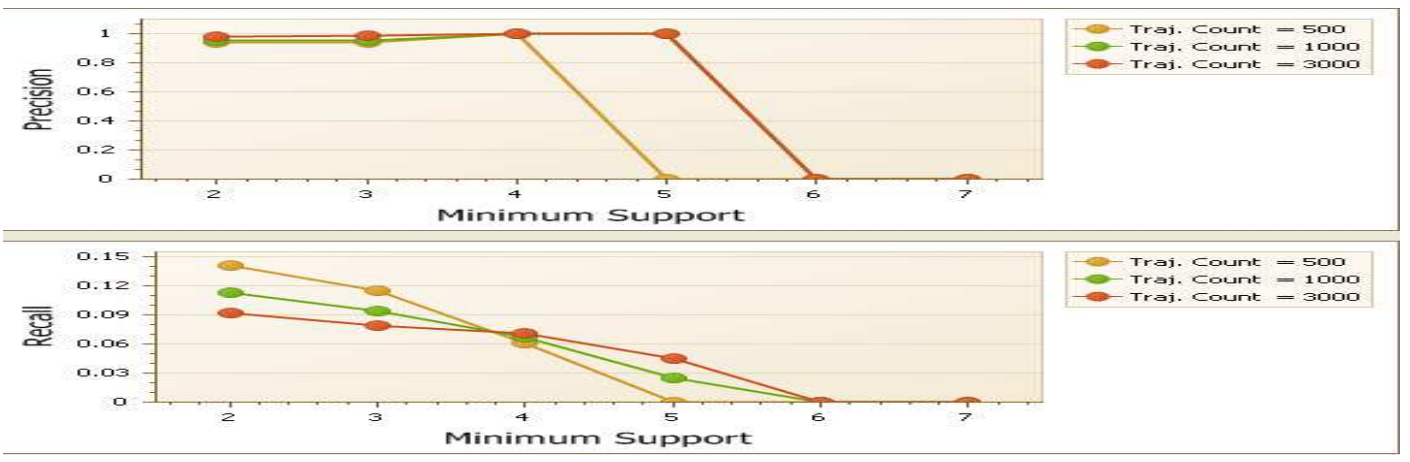

Figure (8) Precision and Recall Vs Min sup Value Using (TR=500, 1000, 3000) trajectories

\subsection{Effect of Minimum confidence}

There is a direct effect of changing the percentage of minimum confidence on the precision and recall percentages of our proposed system. By increasing the min conf value, the percentage of system precision also increased due to the increment in correct predictions compared to total number of predictions occurred. Whereas, recall percentage decreased by increasing min conf value as shown in figure (9). The

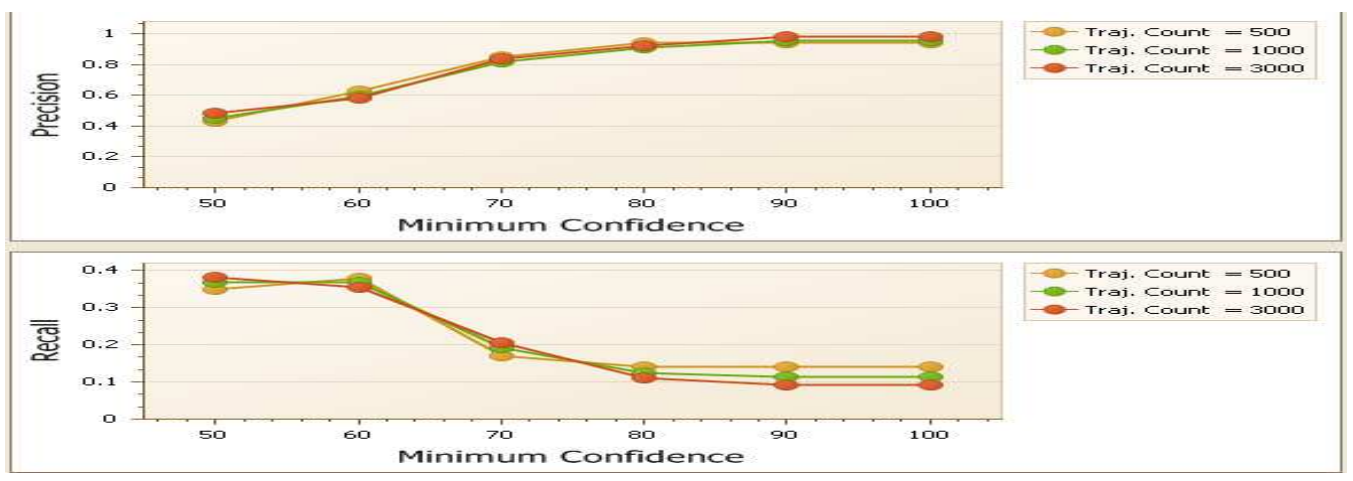

optimum min conf. value $=100 \%$.

Figure (9) Precision and Recall Vs Min conf. Value Using (TR=500 ,1000,3000) trajectories

\subsection{Algorithms' Execution time}

In this section, we study the execution time proprieties of our proposed algorithms to estimate the performance of proposed system. Figure (10) shows execution time measured in $(\mathrm{msec})$ as a function of total number of input trajectories using CTC, STS and intra-sector algorithms. 


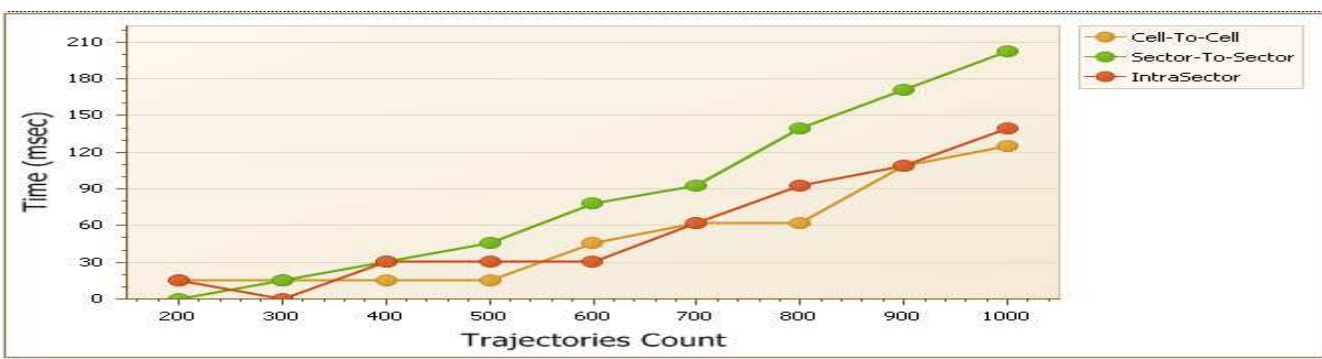

Figure (10) Execution Time in (msec) as a Function of Different Trajectories Length

\subsection{ICMP algorithm among other algorithms}

As RLP algorithm outperformed UMP and GSP algorithm as proved in [8]. ICMP algorithm is mainly using RLP main structure with modifications to be applicable on mobile network platform.

The proposed algorithm is mainly considered as the most practical one which can be applied on mobile users at any environment due to its flexibility and sub algorithms are adapted to suite various location based applications in addition to enhancement of mobile network allocation resources and mobility management.

ICMP proposed algorithm benefits from U-TDOA technique to introduce sector to sector and intra sector predictions. These techniques are highly efficient in LBS applications based on mobile networks. The optimum parameters and working modes can be extracted depending on the needed application, user environments and flexibility of adjusting input data parameters.

The proposed algorithm not only theoretical one but it represent practical solution for future location prediction based on mobile network platform at various environments.

\section{Conclusion}

According to the improvements in positioning techniques based on mobile network platform in addition to the increasing demand on LBS applications, developed algorithms used in future prediction of mobile users represent effective part in improving services provided by mobile network operators. Future location prediction is highly effective to enhance mobile network allocation resources, hand over process and mobility management operations.

U_TDOA technique on UMTS network platform is the used technique for accurate location detection specially while using intra-sector prediction level which will give high position accuracy.

Using mobile network platform is suitable for millions of people all over the world with lower costs than using GPS technology in addition that it can be used at indoor or outdoor areas.

In this paper, a new algorithm (ICMP) depending on (RLP) fundamentals with modification to agreed with mobile network requirements is developed.

ICMP algorithm is divided into three main parts cell to cell, sector to sector and intra-sector levels of predictions, each prediction level is convenient to specific application within certain environment. 
The main steps used in the proposed prediction method begin with map reconstruction to specific hexagonal cells and sectors. Then, database should be implemented depending on the last recorded movement of users and according to the used sub pattern needed to be used. After that, moving sequences are created according to algorithm restrictions and frequent movement patterns are obtained according to ICMP suggestions. Finally rules are extracted and next movements are predicted. ICMP algorithm shows high obtained results with valuable precision and recall values within good execution time. The proposed method can be adapted according to the needed application and the surrounding environments.

Now we are looking forward to enhance mobile network positioning methods to be with acceptable and comparable accuracy with other used positioning techniques. Moreover, we are working to develop proposed algorithm to enhance accuracy, execution time and to make relationship between the currently used technique and the modern positioning techniques like GIS systems to share their advantages.

\section{References}

[1] R. Agrawal, R. Srikant, "Mining sequential patterns", Proceedings of the IEEE Conference on Data Engineering, ICDE_95, pp. 487-499, 1995.

[2] G.Y. Liu, M.Q. Gerald, "A predictive mobility management algorithm for wireless mobile computing and communications", Proceedings of the IEEE International Conference on Universal Personal Communications, pp. 268-272, 1995.

[3] T. Liu, P. Bahl, I. Chlamtac, "Mobility modeling, location tracking, and trajectory prediction in wireless ATM networks", IEEE J. Select, 1998.

[4] I.F. Akyildiz, S.M. Ho,Y.-B. Lin, " Movement-based location update and selective paging for PCS networks", IEEEACM Trans. Network,1996.

[5] S. Rajagopal, R.B. Srinivasan, R.B. Narayan, X.B.C. Petit," GPS-based predictive resource allocation in cellular networks" , Proceedings of the IEEE International Conference on Networks (IEEE ICON_02), 2002.

[6] D. Katsaros, A. Nanopoulos, M. Karakaya, G. Yavas, O. Ulusoy, Y. Manolopoulos," Clustering mobile trajectories for resource allocation in mobile environments" , Intelligent Data Analysis Conference (IDA_2003)Lecture Notes in Computer Science, pp. 319-329, 2003.

[7]Go khan Yavas, Dimitrios Katsaros, O. zgu r Ulusoy, Yannis Manolopoulos ," A data mining approach for location prediction in mobile environments", pp. 121146, 2004.

[8] Thi Hong Nhan Vu , Keun Ho Ryu , Namkyu Park , " A method for predicting future location of mobile user for location-based services system", Computers \& Industrial Engineering 57 , pp. 91-105, 2009.

[9] Axel K"upper," Location based services fundamentals and operation", John Wiley \& Sons Ltd, The Atrium, Southern Gate, Chichester, West Sussex PO19 8SQ, England, 2005.

[10] " TSG-RAN Meeting \#24 RP-040164", Seoul, Korea 2-4 June 2004.

[11] Javid Taheri, Albert Y. Zomaya , " Clustering techniques for dynamic location management in mobile computing", 2007. 
التنبؤ بمواقع مستخدمي الهاتف الخلوي باستخدام نموذج الحركة داخل خلية شبكة المحمول في شبكة النظام العالمي للاتصالات النلاسلكية الهولية

تعد خدمات تحديد المواقع وتطبيقاتها (LBS) من أكثر الخدمات المتتامية لنظم الاتصالات اللاسلكية. التتبؤ

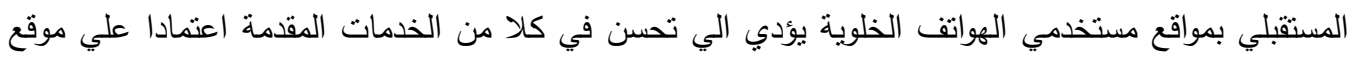
المستخدم والخدمات المعتمدة علي أداء الثبكة. في الدوريات العلمية تم نشر العديد من البحوث لتطوير طرق

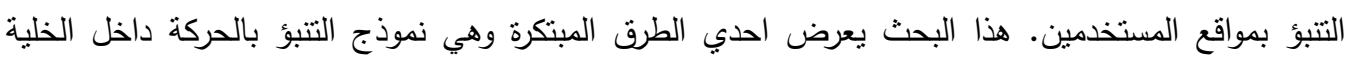
لالتتبؤ بمواقع مستخدمي الهاتق الخلوي في شبكة النظام العالمي للاتصالات اللاسلكية (UMTS) (ICMP)

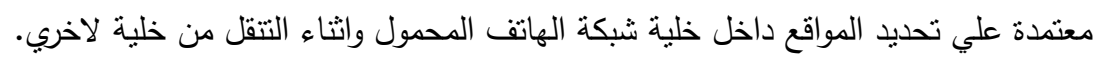

هذه الطريقة تحتمد علي تكوين قاعدة بيانات لمستخدمي الثبكة بالاضافة الي توظيف طرق تحديد المواقع

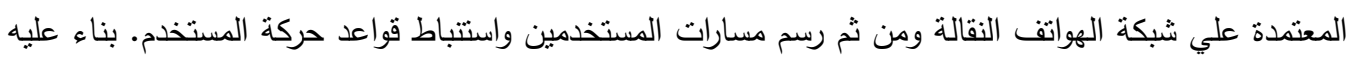

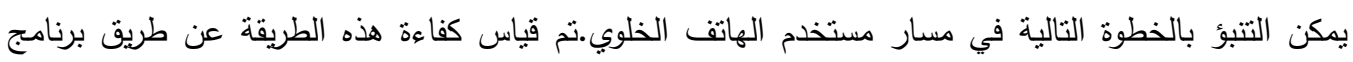

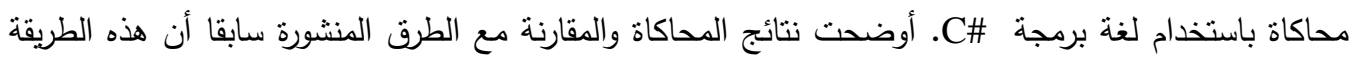

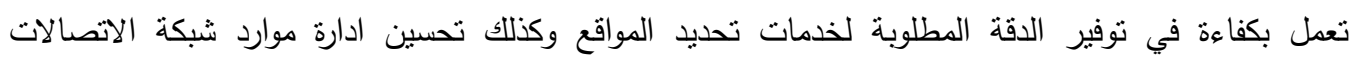

